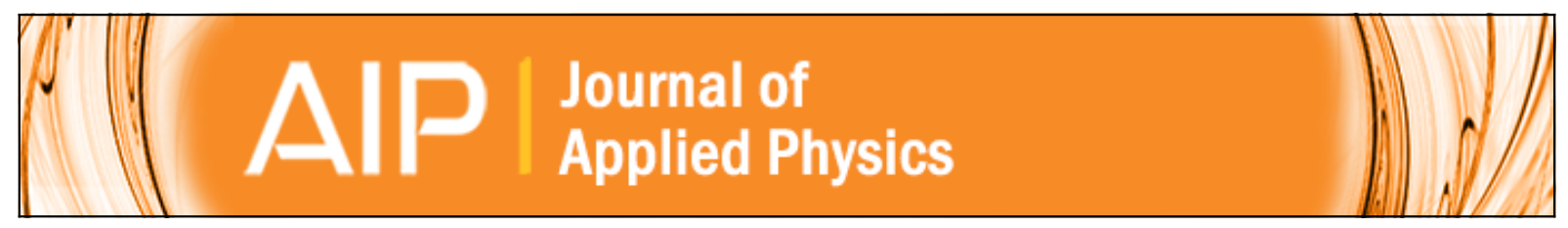

\title{
Violet and blue light amplification in Nd 3+-doped fluoroindate glasses
}

G. S. Maciel, L. de S. Menezes, Cid B. de Araújo, and Y. Messaddeq

Citation: Journal of Applied Physics 85, 6782 (1999); doi: 10.1063/1.370194

View online: http://dx.doi.org/10.1063/1.370194

View Table of Contents: http://scitation.aip.org/content/aip/journal/jap/85/9?ver=pdfcov

Published by the AIP Publishing

\section{Articles you may be interested in}

Blue upconversion enhancement by a factor of 200 in Tm 3+-doped tellurite glass by codoping with $\mathrm{Nd} 3+$ ions J. Appl. Phys. 92, 6337 (2002); 10.1063/1.1515376

Frequency upconversion involving triads and quartets of ions in a $\operatorname{Pr} 3+$ / Nd 3+ codoped fluoroindate glass J. Appl. Phys. 92, 3065 (2002); 10.1063/1.1501757

Blue up-conversion luminescence and energy transfer process in Nd 3+ - Yb 3+ - Tm 3+ co-doped ZrF 4 -based glasses

J. Appl. Phys. 91, 954 (2002); 10.1063/1.1428805

Thermally enhanced frequency upconversion in $\mathrm{Nd} 3+-$ doped fluoroindate glass

J. Appl. Phys. 90, 4498 (2001); 10.1063/1.1410326

Simultaneous generation of red, green, and blue continuous-wave laser radiation in $\mathrm{Nd} \mathrm{3+-doped} \mathrm{aperiodically}$ poled lithium niobate

Appl. Phys. Lett. 78, 144 (2001); 10.1063/1.1338495

\section{AlP Re-register for Table of Content Alerts}

\section{Create a profile.

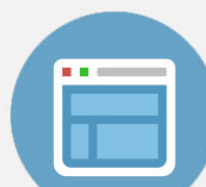 \\ Sign up today!}




\title{
Violet and blue light amplification in $\mathrm{Nd}^{3+}$-doped fluoroindate glasses
}

\author{
G. S. Maciel, L. de S. Menezes, and Cid B. de Araújo \\ Núcleo de Excelência em Óptica Não Linear, Lasers e Aplicações-NEON, Departamento de Física, \\ Universidade Federal de Pernambuco, 50670-901 Recife, PE, Brazil \\ Y. Messaddeq \\ Instituto de Química, Universidade do Estado de São Paulo, 14800-900 Araraquara, SP, Brazil
}

(Received 22 September 1998; accepted for publication 3 February 1999)

\begin{abstract}
We report optical gain at 382 and $414 \mathrm{~nm}$ from $\mathrm{Nd}^{3+}$-doped fluroindate glasses after excitation with lasers operating either at 583 or $532 \mathrm{~nm}$. Stimulated emission due to a frequency upconversion process results in increase of the emitted violet and blue light intensity and emission line narrowing. Large optical gain is measured by pump-probe spectroscopy using samples with various $\mathrm{Nd}^{3+}$ concentrations. (c) 1999 American Institute of Physics. [S0021-8979(99)09109-4]
\end{abstract}

\section{INTRODUCTION}

Solid-state lasers based on frequency upconversion phenomena in rare-earth (RE) doped materials ${ }^{1-7}$ are promising violet/blue light sources for applications such as high-density data storage, displays, contaminant detection, and biological research. $^{8}$

A common procedure to investigate new upconversion lasers is to choose a convenient host with a low energy phonon spectrum which allows a larger lifetime of RE metastable states and large luminescence efficiency. Amongst the suitable hosts such as heavy metal-based amorphous matrices, fluoride glasses have received great attention due to its small optical phonon energies. ${ }^{7}$ Fluoroindate glasses (FIGs) belong to this class of materials and they have been extensively studied in the last years. ${ }^{9-20}$ Besides a large efficiency as frequency upconverters, FIGs are known to have a high resistance to moisture with good chemical and mechanical stability and a large transparency window $(0.2-8 \mu \mathrm{m})$.

Up to the present upconversion laser performance has been evaluated through laser slope-efficiency measurements and not much attention has been devoted to measure the nonlinear coefficient related to the mechanism responsible for feeding population to the fluorescent levels. This is generally difficult to quantify because the amplification of the signal in one-pass events in the nonlinear medium is usually small.

In this article we report the first observation of amplification in violet and blue wavelengths using $\mathrm{Nd}^{3+}$-doped FIG samples excited by lasers operating in the red/green range. A method to measure the nonlinear coefficient, using an incoherent light source as a probe and a laser beam as the pump source, is introduced based upon the behavior of Fourier components of the amplified signal. The results are presented for two different pumping wavelengths and various $\mathrm{Nd}^{3+}$ concentrations. Comparison with results obtained for $\mathrm{Nd}^{3+}$-doped fluorozirconate (ZBLAN) glass is also made.

\section{EXPERIMENTAL SETUP AND TECHNIQUE USED}

The fluoroindate glasses used were based on $\mathrm{InF}_{3}$ as the network former and various modifiers. The glass synthesis was implemented using the procedure given in Ref. 9 and the actual composition used in the present experiments is the following in mole percent: (39- $x$ ) $\mathrm{InF}_{3}-20 \mathrm{ZnF}_{2}-20 \mathrm{SrF}_{2}-16$ $\mathrm{BaF}_{2}-2 \mathrm{GdF}_{3}-2 \mathrm{NaF}-1 \mathrm{GaF}_{3}-x \mathrm{NdF}_{3}(x=0.5,1,3)$. One sample of ZBLAN glass with the following composition in mole percent: $52 \mathrm{ZrF}_{4}-20 \mathrm{NaF}-20 \mathrm{BaF}_{2}-4 \mathrm{LaF}_{3}-3 \mathrm{AlF}_{3}-1 \mathrm{NdF}_{3}$ was also used for comparison with FIG samples. Linear absorption measurements were made using a commercial spectrophotometer.

Figure 1 is the schematic of the experimental setup for nonlinear measurements. The probe beam was obtained using a low power white lamp whose wavelength $\left(\lambda_{1}\right)$ was selected using a $25 \mathrm{~cm}$ grating monochromator. The pump beam at wavelength $\lambda_{2}$ was obtained from a dye laser pumped by the second harmonic of a neodymium:yttriumaluminum-garnet laser or the second harmonic beam. A mechanical chopper was used to modulate the intensity of the probe and pump beams at the frequencies $f_{1}$ and $f_{2}$, respectively. The signals were detected by a GaAs photomultiplier connected to a lock-in amplifier, which is triggered by an electronic signal generated by the chopper. The detection technique used was based on the measurements of the Fourier component at $\left(f_{1}+f_{2}\right)$ present in the transmitted probe beam due to the presence of the pump beam.

The propagation of the probe beam with intensity $I_{1}$ through the nonlinear medium excited by the strong pump laser beam with intensity $I_{2}$, can be described by

$$
\frac{d I_{1}(z)}{d z}=-\alpha_{1} I_{1}(z)+\beta_{12} I_{2}^{2}(z) I_{1}(z)
$$

The solution of Eq. (1) for the transmitted intensity is given by

$$
\begin{aligned}
I_{1}(d)= & (1-R)^{2} \exp \left(-\alpha_{1} d\right) I_{1}(0) \exp \left\{\beta_{12}(1-R)^{2} I_{2}^{2}(0)\right. \\
& \left.\left.\times\left[1-\exp \left(-2 \alpha_{2} d\right)\right] / 2 \alpha_{2}\right)\right\},
\end{aligned}
$$

where $\alpha_{1}$ and $\alpha_{2}$ are the linear absorption coefficients at the probe and pump beam wavelengths, respectively, $R$ is the reflectivity at the sample's surfaces, $d$ is the sample thickness, $I_{2}(0)$ is the intensity of the pump beam just before entering the sample and $\beta_{12}$ is the nonlinear coefficient. The 


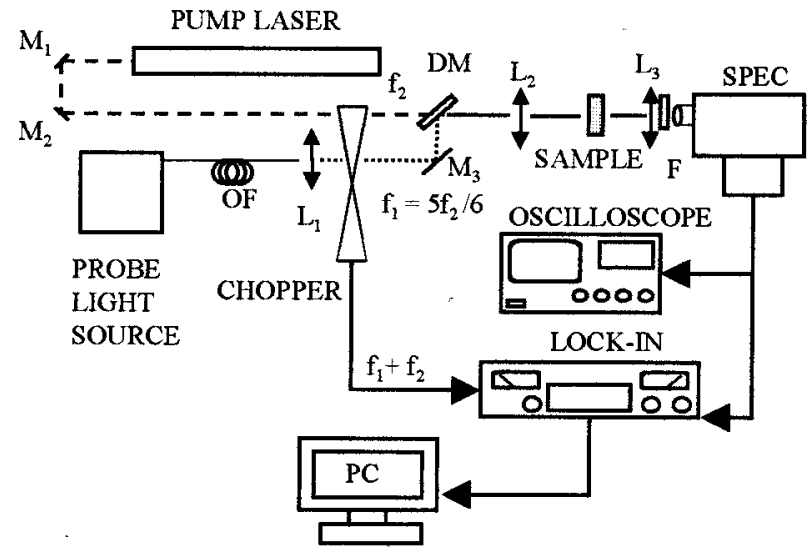

FIG. 1. Experimental setup used for the nonlinear measurements. $M_{1}, M_{2}$, and $\mathrm{M}_{3}$ are metallic mirrors; $\mathrm{DM}$ is a dichroic mirror; $\mathrm{L}_{1}, \mathrm{~L}_{2}$, and $\mathrm{L}_{3}$ are convex lenses of focal length equal to 5,5 , and $10 \mathrm{~cm}$, respectively; $\mathrm{F}$ are color filters, OF is a multimode optical fiber, SPEC is the monochromator and the photomultiplier tube.

nonlinear term in Eq. (1) arises because the intensity of the probe beam is affected by the presence of the pump beam, and the quadratic dependence on $I_{2}$ comes from the fact that two photons are necessary to originate the nonlinearity as will be explained below. The value of $\beta_{12}$ is usually small and the intensity change of the signal due to its passage throughout the sample is feeble. The main difficulty in this kind of experiments is to distinguish between the spontaneous emission generated at $\lambda_{1}$ due the frequency upconversion process excited by the pump beam and the amplified probe beam component. As shown in Fig. 1, the strong beam is made collinear with the probe beam and both beams are focused on the sample. A broad band incoherent light source was used as the probe beam and the spectral region of interest $(360-440 \mathrm{~nm})$ to be sent through the sample is selected using a dichroic mirror (DM). The experiments were performed at two wavelengths of the pump beam at 583 or 532 $\mathrm{nm}$. The pump and probe beams were chopped at different frequencies that are not harmonic of each other before they reach the DM. As indicated in Fig. 1 we choose $f_{1}=5 f_{2} / 6$ with $f_{2}=112 \mathrm{~Hz}$. Color filters at the entrance of the spectrometer prevent scattered light due to the pump beam to enter the monochromator. The light signal from the sample, in the violet-blue range, has three components: an upconverted scattered light modulated at frequency $f_{2}$, the probe beam with a frequency modulation $f_{1}$, and a component with frequency $\left(f_{1}+f_{2}\right)$ that results from the interaction of both beams, $I_{1}$ and $I_{2}$, with the $\mathrm{Nd}^{3+}$ ions in the sample. The signal at $\left(f_{1}+f_{2}\right)$ carrying information on the stimulated emission process is electronically filtered by a lock in and the data are sent to a personal computer for processing.

\section{RESULTS AND DISCUSSIONS}

The linear absorption spectrum of one sample is shown in Fig. 2(a). The bands are due to the $\mathrm{Nd}^{3+}$ ions and their positions are independent of the $\mathrm{Nd}^{3+}$ concentration. This is characteristic of inhomogeneously broadened transitions inside the $4 f$ shell which are not very sensitive to the perturbing crystalline field.
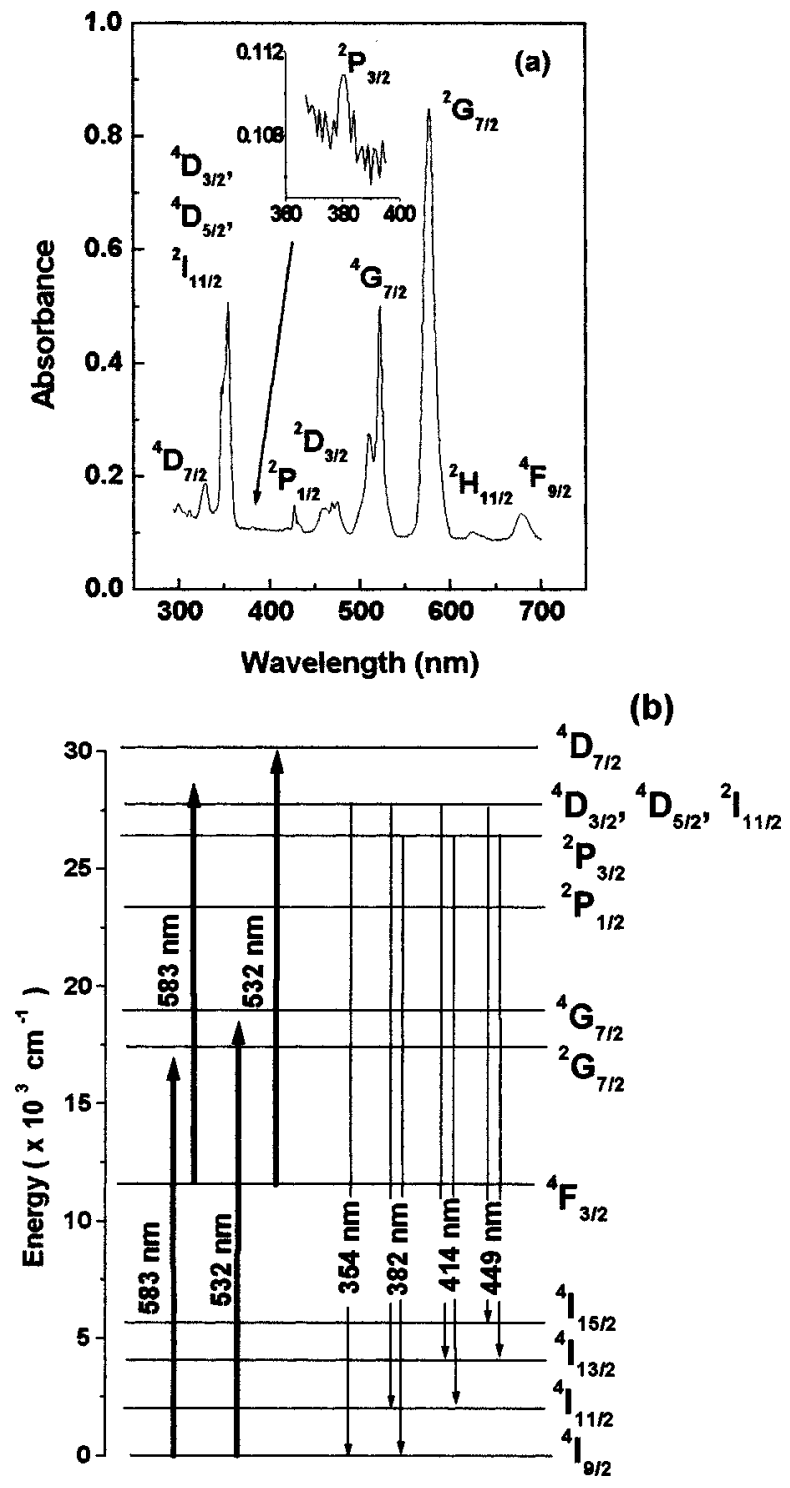

FIG. 2. (a) Absorbance spectrum of $\mathrm{Nd}^{3+}$-doped fluoroindate glass (sample with $x=3$ and thickness $d=1.80 \mathrm{~mm}$ ). The absorption band at $380 \mathrm{~nm}$ $\left({ }^{2} P_{3 / 2}\right)$ is magnified for visualization; (b) simplified energy level scheme showing the excitation pathways and the observed upconversion fluorescence emissions.

In one set of experiments the pumping wavelength was tuned to $583 \mathrm{~nm}$, close to the absorption band centered at $577 \mathrm{~nm}\left({ }^{4} I_{9 / 2} \rightarrow{ }^{2} G_{7 / 2}\right)$ but not on resonance to avoid hole burning due to the large power emitted by the dye laser. Upconverted fluorescent lines were observed at wavelengths $354 \mathrm{~nm} \quad\left({ }^{4} D_{3 / 2}, \quad{ }^{4} D_{5 / 2}, \quad{ }^{2} I_{11 / 2} \rightarrow{ }^{4} I_{9 / 2}\right), \quad 382 \mathrm{~nm} \quad\left({ }^{2} P_{3 / 2}\right.$ $\left.\rightarrow{ }^{4} I_{9 / 2} ; \quad{ }^{4} D_{3 / 2}, \quad{ }^{4} D_{5 / 2}, \quad{ }^{2} I_{11 / 2} \rightarrow{ }^{4} I_{11 / 2}\right), \quad 414 \mathrm{~nm} \quad\left({ }^{2} P_{3 / 2}\right.$ $\left.\rightarrow{ }^{4} I_{11 / 2} ;{ }^{4} D_{3 / 2},{ }^{4} D_{5 / 2},{ }^{2} I_{11 / 2} \rightarrow{ }^{4} I_{13 / 2}\right)$, and $449 \mathrm{~nm}\left({ }^{2} P_{3 / 2}\right.$ $\left.\rightarrow{ }^{4} I_{13 / 2} ;{ }^{4} D_{3 / 2},{ }^{4} D_{5 / 2},{ }^{2} I_{11 / 2} \rightarrow{ }^{4} I_{15 / 2}\right)$, corresponding to two photons absorbed from the pump beam as indicated in Fig. 2(b). Other emissions in the visible and in the infrared range can be easily assigned to the $\mathrm{Nd}^{3+}$ levels but we are not interested in their behavior for the present purpose. A log$\log$ plot of the violet and blue fluorescence as a function of the pump power exhibited a quadratic dependence for each emission line, corroborating the assumption that two photons from the excitation source are necessary to excite levels 

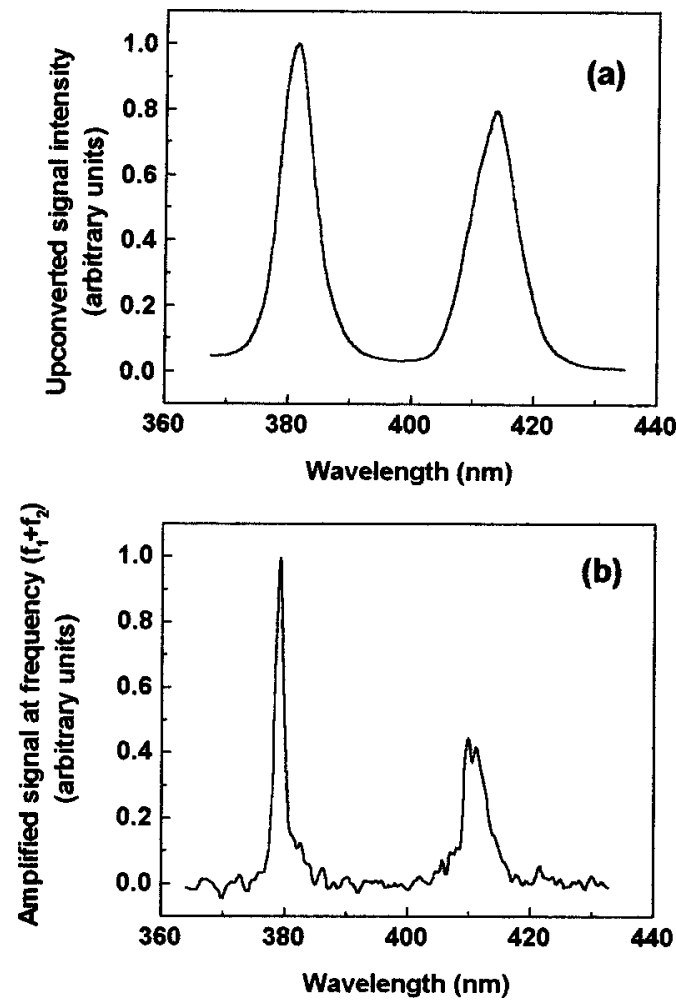

FIG. 3. (a) Upconverted emission lines at 382 and $414 \mathrm{~nm}$ for excitation at $583 \mathrm{~nm}$ in FIG sample with $x=0.5$; (b) probe beam amplified signal modulated at $\left(f_{1}+f_{2}\right)$ for the sample with $x=0.5$. The probe wavelength $\left(\lambda_{1}\right)$ was scanned in the range between 370 and $430 \mathrm{~nm}$ while the excitation wavelength $\left(\lambda_{2}\right)$ was fixed at $583 \mathrm{~nm}$. The scales are arbitrary.

${ }^{4} D_{3 / 2},{ }^{4} D_{5 / 2},{ }^{2} I_{11 / 2}$, and ${ }^{2} P_{3 / 2}$ and to produce each upconverted photon. The spectrum corresponding to emissions at 382 and $414 \mathrm{~nm}$ are shown in Fig. 3(a) for the sample with $x=0.5$. These emissions have been previously studied under pulsed $^{19}$ and continuous wave excitation ${ }^{20}$ and they are particularly interesting because of their large oscillator strengths. Since it was already observed laser action in $\mathrm{Nd}^{3+}$-doped ZBLAN fibers at these wavelengths, ${ }^{21,22}$ we set an apparatus to measure the coefficient $\beta_{12}$ in our samples and to investigate the expected stimulated emission when the pump and probe beams are present.

After a careful adjustment of the overlap between the probe and the pump beams on the sample we scanned the probe beam wavelength and recorded the signal at $\left(f_{1}+f_{2}\right)$ for a fixed incident pump power. In this case the signals at $\left(f_{1}+f_{2}\right)$ are observed only at two wavelengths correspond-
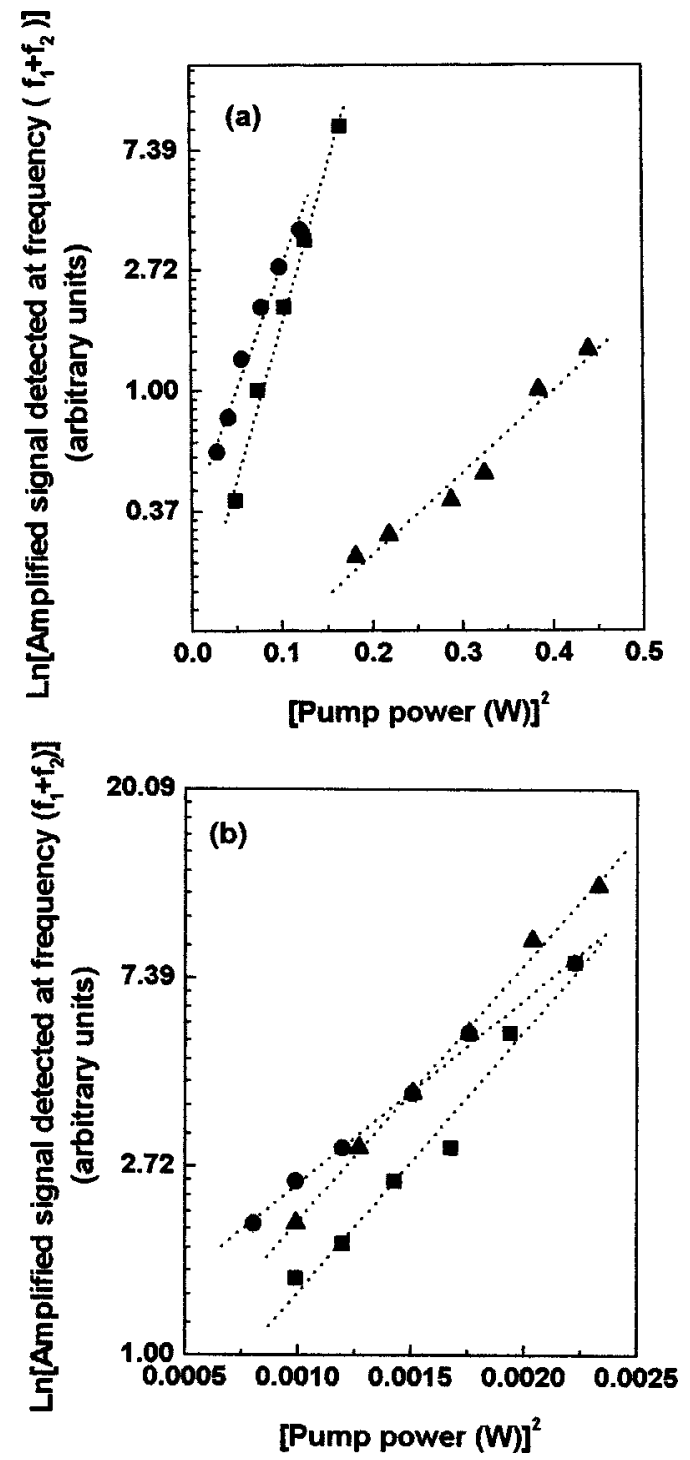

FIG. 4. (a) Signal at $414 \mathrm{~nm}$ detected at frequency $\left(f_{1}+f_{2}\right)$ as a function of the squared pump power for excitation at $532 \mathrm{~nm}$ in FIG sample with $x$ $=1$ and thickness $d=1.45 \mathrm{~mm}(\mathbf{\square}) ; x=3$ and $d=1.80 \mathrm{~mm}(\boldsymbol{O})$; and ZBLAN with $x=1$ and $d=2.45 \mathrm{~mm}(\mathbf{\Lambda})$. (b) Same as in (a) with pump wavelength at $583 \mathrm{~nm}$. The dashed lines represent the fit of the experimental data with Eq. (2) with the coefficients $\beta_{12}$ of Table I.

ing to the upconversion lines at 382 and $414 \mathrm{~nm}$ as illustrated in Fig. 3(b). We also measured the amplitude of the signal modulated at $\left(f_{1}+f_{2}\right)$ as a function of the pump power for various $\mathrm{Nd}^{3+}$ concentrations and typical results are shown in

TABLE I. Values obtained for the nonlinear coefficient $\beta_{12}\left(\mathrm{~cm}^{3} / \mathrm{W}^{2}\right)$ using different excitation wavelengths and different concentrations of $\mathrm{Nd}^{3+}$ in FIG and ZBLAN.

\begin{tabular}{|c|c|c|c|c|c|}
\hline \multicolumn{2}{|c|}{ Pump wavelength } & \multicolumn{2}{|c|}{$532 \mathrm{~nm}$} & \multicolumn{2}{|c|}{$583 \mathrm{~nm}$} \\
\hline $\begin{array}{l}\text { Sample } \\
\text { \Emissior }\end{array}$ & & $382 \mathrm{~nm}$ & $414 \mathrm{~nm}$ & $382 \mathrm{~nm}$ & $414 \mathrm{~nm}$ \\
\hline FIG & $\begin{array}{c}x=0.5 \\
x=1 \\
x=3\end{array}$ & $\begin{array}{l}(1.9 \pm 0.1) \times 10^{-11} \\
(5.3 \pm 0.2) \times 10^{-11} \\
(3.4 \pm 0.1) \times 10^{-11}\end{array}$ & $\begin{array}{l}(1.1 \pm 0.1) \times 10^{-11} \\
(1.8 \pm 0.1) \times 10^{-11} \\
(1.3 \pm 0.1) \times 10^{-11}\end{array}$ & $\begin{array}{l}(2.6 \pm 0.1) \times 10^{-7} \\
(6.3 \pm 0.2) \times 10^{-7} \\
(4.4 \pm 0.3) \times 10^{-7}\end{array}$ & $\begin{array}{l}(2.0 \pm 0.1) \times 10^{-7} \\
(3.2 \pm 0.2) \times 10^{-7} \\
(3.1 \pm 0.1) \times 10^{-7}\end{array}$ \\
\hline ZBLAN & $x=1$ & $(2.3 \pm 0.3) \times 10^{-12}$ & $(3.3 \pm 0.3) \times 10^{-12}$ & $(2.7 \pm 0.1) \times 10^{-7}$ & $(2.2 \pm 0.1) \times 10^{-7}$ \\
\hline
\end{tabular}


Fig. 4 for the samples with $x=1$ and 3. The measurements were repeated with the pump laser at $532 \mathrm{~nm}$ and both fluorescence lines were analyzed. As shown in Fig. 4 the experimental results exhibit an exponential growth as a function of the pump intensity that is observed for all samples studied. Also shown are the results for $\mathrm{ZBLAN}: \mathrm{Nd}^{3+}$ sample. The exponential curves fitted to the experimental results with basis in Eq. (2) give the values of the nonlinear coefficient $\beta_{12}$ shown in Table I for the two excitation wavelengths and for different concentrations of $\mathrm{Nd}^{3+}$ in both host matrices. It is important to note that the values of $\beta_{12}$ for $583 \mathrm{~nm}$ are $\sim 10^{4}$ times larger than the values obtained for $532 \mathrm{~nm}$. This enhancement occurs because the laser at $583 \mathrm{~nm}$ is closer to resonance and the oscillator strength of the transition peaked at $577 \mathrm{~nm}$ is larger than for the absorption line centered at $521 \mathrm{~nm}$. We also observe that the most efficient sample is the $\mathrm{Nd}^{3+}$-doped fluoroindate glass with $x=1$. The reason being the strong fluorescence quenching in the more concentrated sample with $x=3$ due to cross-relaxation processes between $\mathrm{Nd}^{3+}$ neighbors. ${ }^{19}$

Another relevant feature to consider is the behavior of the linewidths $(\Delta \lambda)$ of the upconverted emissions in Fig. 3(a) in comparison with the spectrum of Fig. 3(b). The value of $\Delta \lambda$ for the amplified emissions is smaller for both lines at 382 and $414 \mathrm{~nm}$. This line narrowing is due to the amplification process even though the interaction length is only 1.80 $\mathrm{mm}$.

The results reported indicate the possibility of using FIG as a better gain medium than ZBLAN at 382 and $414 \mathrm{~nm}$. Of course the development of good quality optical fibers based on FIG may allow the operation of efficient upconversion fiber lasers in the future. ${ }^{23}$

Finally, in order to be sure that the detected signals were due to stimulated emissions instead of induced absorption, an independent experiment was performed. Using two HeNe lasers modulated at frequencies $f_{1}$ and $f_{2}$, we operated the photodetector in the nonlinear regime, set the lock-in phase to $\phi=0$ and measured a negative signal at $\left(f_{1}+f_{2}\right)$. Keeping the lock-in parameters fixed such that $\phi=0$, and returning to the scheme of Fig. 1, we observed a positive signal at $\left(f_{1}\right.$ $+f_{2}$ ) which indicates that a signal due to stimulated emission was detected.

In summary the results presented in this article demonstrate a simple method to measure optical gain in single-pass experiments using bulk samples doped with RE ions. The technique has shown a high sensitivity and allowed us to determine the gain parameter for the violet and blue emissions at 382 and $414 \mathrm{~nm}$ in $\mathrm{Nd}^{3+}$-doped FIG and ZBLAN for two absorption bands pumped either at 583 or $532 \mathrm{~nm}$. The results show that operation of efficient upconversion la- sers based in $\mathrm{Nd}^{3+}$-doped fluoroindate glass fibers exploiting the process studied here is promising.

\section{ACKNOWLEDGMENTS}

This work was partially supported by the Brazilian agencies Conselho Nacional de Desenvolvimento Cientifico e Tecnológico (CNPq), Financiadora Nacional de Estudos e Projetos (FINEP), Fundação Coordenação de Pessoal de Nível Superior (CAPES), and Fundação de Amparo à Ciência e Tecnologia do Estado de Pernambuco (FACEPE). The authors thank J. R. Rios Leite and L. H. Acioli for helpful discussions and N. Rakov for helping with the measurements. They also acknowledge Professor M. Poulain from Université de Rennes (France) for providing us the ZBLAN sample used.

${ }^{1}$ L. F. Johnson and H. G. Guggenheim, Appl. Phys. Lett. 19, 44 (1971).

${ }^{2}$ A. J. Silversmith, W. Lenth, and R. M. Macfarlane, Appl. Phys. Lett. 51, 1977 (1987)

${ }^{3}$ R. M. Macfarlane, F. Tong, A. J. Silversmith, and W. Lenth, Appl. Phys. Lett. 52, 1300 (1988).

${ }^{4}$ P. Xie and S. C. Rand, Opt. Lett. 15, 848 (1990).

${ }^{5}$ P. Xie and S. C. Rand, Appl. Phys. Lett. 57, 1182 (1990).

${ }^{6}$ P. Xie and S. C. Rand, Appl. Phys. Lett. 63, 3125 (1993).

${ }^{7}$ See for example, Rare-Earth Doped Fiber Lasers and Amplifiers, edited by M. J. Digonnet (Dekker, New York, 1993), and references therein.

${ }^{8}$ For many references on the subject see: Proceedings of the International Conference on Luminescence, Prague, 1996 ( J. Lumin. 72-74, (1997)); Proceedings of the Tenth International Symposium on Non-Oxide Glasses, Corning, NY, 1996 ( J. Non-Cryst. Solids 213-214, (1997)); Proceedings of the Third Brazilian Symposium on Glasses and Related Materials, Bonito, 1998 (J. Non-Cryst. Solids-to be published).

${ }^{9}$ L. E. E. de Araújo, A. S. L. Gomes, C. B. de Araújo, Y. Messaddeq, A. Florez, and M. A. Aegerter, Phys. Rev. B 50, 16219 (1994).

${ }^{10}$ G. S. Maciel, C. B. de Araújo, Y. Messaddeq, and M. A. Aegerter, Phys. Rev. B 55, 6335 (1997)

${ }^{11}$ L. de S. Menezes, C. B. de Araújo, G. S. Maciel, and Y. Messaddeq, Appl. Phys. Lett. 70, 683 (1997).

${ }^{12}$ N. Rakov, C. B. de Araújo, Y. Messaddeq, and M. A. Aegerter, Appl. Phys. Lett. 70, 3084 (1997).

${ }^{13}$ W. Lozano, C. B. de Araújo, C. Egalon, A. S. L. Gomes, B. J. Costa, and Y. Messaddeq, Opt. Commun. 153, 271 (1998).

${ }^{14}$ E. M. Pacheco, C. B. de Araújo, and Y. Messaddeq, J. Non-Cryst. Solids 226, 265 (1998).

${ }^{15}$ J. Fernández, R. Bolda, and M. A. Arriandiago, Opt. Mater. 4, 91 (1994).

${ }^{16}$ S. Kishimoto and K. Hirao, J. Appl. Phys. 80, 1965 (1996).

${ }^{17}$ M. Dejneka, E. Snitzer, and R. E. Rimant, J. Lumin. 65, 227 (1995).

${ }^{18}$ S. Tanabe, T. Hanada, M. Watanabe, T. Hayashi, and N. Soga, J. Am. Ceram. Soc. 78, 2917 (1997).

${ }^{19}$ L. de S. Menezes, C. B. de Araújo, Y. Messaddeq, and M. A. Aegerter, J. Non-Cryst. Solids 213/214, 256 (1997).

${ }^{20}$ L. H. Acioli, J.-T. Guo, C. B. de Araújo, Y. Messaddeq, and M. A. Aegerter, J. Lumin. 72-74, 68 (1997).

${ }^{21}$ D. S. Funk, J. W. Carlson, and J. G. Eden, Electron. Lett. 30, 1860 (1994).

${ }^{22}$ D. S. Funk, J. W. Carlson, and J. G. Eden, Opt. Lett. 20, 1474 (1995).

${ }^{23}$ Fibers based on fluoroindate glass presenting low losses have been developed recently by M. Poulain and co-workers at Université de Rennes in France (private communication). 